\title{
Direito à saúde das pessoas LGBTQ+ em privação de liberdade: o que dizem as políticas sociais de saúde no Brasil?
}

Right to health of LGBTQ+ people deprived of freedom: what do social health policies say in Brazil?

Derecho a la salud de las personas LGBTQ+ en privación de libertad: ¿qué dicen las políticas de salud social en Brasil?

\author{
Luís Paulo Souza e Souza ${ }^{1}$ \\ Gabriel Silvestre Minucci² \\ Andrea Matias Alves ${ }^{3}$ \\ Rauni Jandé Roama-Alves ${ }^{4}$ \\ Marconi Moura Fernandes ${ }^{5}$
}

\begin{abstract}
Resumo
Objetivo: debater a realidade da garantia da assistência à saúde de pessoas LGBTQ+ enquanto luta pela solidificação dos direitos humanos frente às políticas públicas de saúde no sistema prisional brasileiro. Metodologia: revisão bibliográfica e documental, analisando livros, artigos e documentos oficiais do governo. Resultados: as pessoas LGBTQ+, minorias políticas, foram, historicamente, colocados à margem dos meios sociais, como família, escola, trabalho, lazer, acesso à justiça e à saúde, encontrando nas vivências periféricas, prostituição e criminalidade, os espaços restantes à abjeção e à negação de seus corpos e estilos/modos de vida. Ao deparar-se com essa população no sistema prisional, esses indivíduos são mantidos sobre as mesmas condições de vulnerabilidade, acrescidas de diversas outras privações instituídas nesse local social. Assim, há a limitação de direitos básicos, destacando-se o direito à saúde, marcas permanentes de desigualdade, marginalização e precariedade de recursos para construção de políticas públicas, apontando uma tripla carga de privação de direitos: direito de ser quem são; direito de estarem onde estão; direito de receberem o que necessitam. Conclusões: no sistema prisional, devem-se considerar aspectos da saúde sexual e reprodutiva, direitos sociais, civis e políticos dessas pessoas. O acesso aos serviços de saúde, tanto nas questões de prevenção quanto nas de tratamento, deve ser assegurado, corroborando com a autonomia dos indivíduos no tocante
\end{abstract}

\footnotetext{
1 Pós-Doutorado em Educação em Saúde, Universidade do Estado do Pará (UEPA); doutor em Saúde Pública, Universidade Federal de Minas Gerais (UFMG); professor adjunto, Faculdade de Medicina, Instituto de Saúde e Biotecnologia (ISB), Universidade Federal do Amazonas (UFAM), Coari, Amazonas, Brasil. http://orcid.org/0000-00029801-4157. E-mail: luis.pauloss@hotmail.com

2 Graduando em Medicina, Universidade Federal de São João del-Rei (UFSJ), campus Dom Bosco, São João del-Rei, Minas Gerais, Brasil. http://orcid.org/0000-0001-5446-647X. E-mail: gabrielsilcci@gmail.com

${ }^{3}$ Especialista em Direito Sanitário e em Saúde Mental, Escola de Saúde Pública do Estado de Minas Gerais (ESPMG); assistente social, Defensoria Pública do Estado de Minas Gerais (DPMG), Belo Horizonte, Minas Gerais, Brasil. http://orcid.org/0000-0002-9427-4883. E-mail: andrea.alves@defensoria.mg.def.br

${ }^{4}$ Doutor em Psicologia; professor adjunto, Departamento de Psicologia, Universidade Federal de Rondonópolis (UFR), Rondonópolis, Mato Grosso, Brasil. http://orcid.org/0000-0002-1982-1488. E-mail: rauniroama@gmail.com

${ }^{5}$ Mestre em Enfermagem e Saúde, Universidade Federal de Minas Gerais (UFMG); psicólogo clínico; professor credenciado, Escola de Saúde Pública do Estado de Minas Gerais (ESPMG), Belo Horizonte, Minas Gerais, Brasil. http://orcid.org/0000-0001-5169-2463. E-mail: marconimf@yahoo.com.br
} 
à vivência da sua sexualidade e de seu gênero, sem discriminação, negligência ou violência. Assegurar esses aspectos é entender que um princípio fundamental do Estado é, sobretudo, fazer viver.

Palavras-chave: Minorias sexuais e de gênero. Direito à saúde. Instituições penais.

\begin{abstract}
Objective: discuss the reality of ensuring the health care of LGBTQ + people while striving for solidification of human rights against public health policies in the brazilian prison system. Methodology: theoretical research, analyzing books, articles and official documents of the government. Results: LGBTQ+ people, political minorities, have historically been placed on the sidelines of social media, such as family, school, work, leisure, access to justice and health, finding in peripheral experiences, prostitution and crime, the remaining spaces to abjection and the denial of their bodies and styles/ways of life. When we encounter this Population in the prison system, even institutionalized-understate protection, these subjects continue to be suffering, with their bodies and souls vulnerable and vulnerability. Thus, in extra environments as intrentrals, there is limitation of basic rights, highlighting the right to health, permanent marks of inequality, marginalization and precariousness of resources for the construction of public policies, pointing a triple burden of deprivation of rights: right to be who they are; right to be where they are; right to receive what they need. Conclusion: in the prison system, aspects of sexual and reproductive health, social, civil and political rights these people should be considered. Access to health services, both in prevention and treatment issues, should be ensured, as well as to ensure the autonomy individuals in relation to the experience of their sexuality and gender, without discrimination, neglect and violence. To ensure these aspects is to understand that a fundamental principle of the State is, aboveall, to live.
\end{abstract}

Keywords: Sexual and gender minorities. Right to health. Prisons.

\title{
Resumen
}

Objetivo: discutir la realidad de garantizar la atención sanitaria de las personas LGBTQ+ mientras lucha por la solidificación de los derechos humanos contra las políticas de salud pública en el sistema penitenciario brasileño. Metodología: investigación teórica, análisis de libros, artículos y documentos oficiales del gobierno. Resultados: las personas LGBTQ+, minorías políticas, históricamente se han puesto al margen de las redes sociales, como la familia, la escuela, el trabajo, el ocio, el acceso a lajusticia y la salud, la búsqueda de experiencias periféricas, la prostitución y lo crimen, los espacios restantes a la objeción y la negación de sus cuerpos y estilos/formas de vida. Cuando nos encontramos cones población el sistema penitenciario, incluso institucionalizado bajo protección estatal, estos sujetos siguen sufriendo, con sus cuerpos y almas vulnerables y vulnerabilizados. Así que, dentro y fuera de las prisiones, existe una limitación de los derechos básicos, destacando el derecho a lasalud, las marcas permanentes de desigualdad, la marginación y la precariedad de los recursos para la construcción de políticas públicas, señalando una triple carga de privación de derechos: Derecho a ser quienes son; Derecho a estar donde estan; Derecho a recibirlo que necesitan. Conclusión: en El sistema penitenciario, deben considerarse aspectos de la salud sexual y reproductiva, los derechos sociales, civiles y políticos de estas personas. Debegarantizarseelacceso a losservicios de salud, tanto enmateria de prevención como de tratamiento, así como garantizarlaautonomía de las personas enrelaciónconlaexperiencia de susexualidad y su género, sindiscriminación, negligencia o violencia. Garantizar estos aspectos es entender que un principio fundamental del Estado es, sobre todo, te hacen vivir. 
Palabras clave: Minorías sexuales y de género. Derecho a la salud. Prisiones.

\section{Introdução}

A existência de minorias políticas LGBTQ+ (lésbicas, gays, bissexuais, travestis, transgêneros, queer e demais sujeitos, incluídos não-binários e assexuais) coloca em xeque as naturalizações sexuais e de gênero e é, ainda, atravessada por outras questões sociais (raciais, econômicas, culturais, etc.). Esse grupo populacional, que historicamente é colocado à margem dos meios sociais, tais como família, trabalho, acesso à justiça e à saúde, encontra nas vivências periféricas, prostituição e criminalidade, os espaços restantes à abjeção e à negação de seus corpos e estilos/modos de vida $(1,2)$. O que se tem imbricado a essas questões é basicamente a continuidade de um processo de subjugação e controle (3). Desse modo, indaga-se:

Afinal, o que é um crime, quando o próprio modo de funcionamento da justiça se faz inseparável de um projeto de atualização perpétua da injustiça como fantasia de controle? O que é um crime, quando tudo o que se entende sob o guarda-chuva da normalidade e legalidade não cessa de reperformar a presença da morte como expectativa de vida de comunidades inteiras, de gente daqui e de toda a terra, humanas e não-humanas? (3)

O segmento populacional LGBTQ+ é marcado pela invisibilidade e por inúmeras barreiras sociais. Toda existência que foge à heteronormatividade compulsória (matrizes heterossexuais, familiares e reprodutivas que pairam sobre a sociedade), segundo a teórica Judith Butler (4), torna-se abjeto, isto é, em uma posição de não-sujeito. Quando um ser nasce, a ele é dado um sexo biológico para o qual obrigatoriamente está a definição de gênero e, dados os construtos sociais, também a sexualidade. Sendo assim, espera-se dos indivíduos comportamentos heterossexuais, ou seja, que, suas relações afetivas se deem com o sexo oposto. Tudo o que foge a isso, aos padrões impostos e à norma, portanto, está fora daquilo que obrigatoriamente se almeja. A norma é um mecanismo regulador dos comportamentos na sociedade, produzindo sujeitos dentro de um padrão comum e de um ideal em relação a todo e qualquer fenômeno. Assim, os corpos LGBTQ+, que contrariam esses domínios sociais, são negados, silenciados, invisibilizados, ausentes de lugar de direitos, colocados como ininteligíveis.

Os indivíduos LGBTQ+ no sistema prisional tornam-se objetos de vulnerabilidade em todas as ordens, individual, social e programática. A vulnerabilidade individual, que compreende os aspectos como comportamento e situação psico-emocional, mostra-se nas 
ações e sofrimentos psíquicos frutos da violência e negação impostas sobre eles. A vulnerabilidade social, que é analisada por normas e suportes sociais, dá-se na privação de recursos sociais e vivências. E a dimensão programática é pensada na forma que as instituições reproduzem ou até mesmo aprofundam as vulnerabilidades vividas por essas pessoas, visível na restrição de direitos e acessos tanto em ambiente intra como extraprisional (5). Ao deparar-se com essa população no sistema prisional, percebe-se que mesmo institucionalizados, sob tutela do estado, os sujeitos LGBTQ+ permanecem e aprofundam em situações de padecimento, sob condições de inúmeras vulnerabilidades de seus corpos e almas $(4,6)$.

Nesse aspecto, o sistema prisional, além de sua constituição judicial, também se coloca enquanto instrumento político na construção de uma criminalidade marcada por questões de desigualdade, marginalização social e econômica, e impossibilidade de acesso à justiça. Sob esse aspecto, o perfil populacional dos indivíduos nesse sistema caracterizase pela repressão e criminalização da pobreza e dos sujeitos periféricos aos interesses e normas (7).

Quando privados de liberdade, os indivíduos levam consigo o estigma permanente de criminoso(a) e são sistemática e intencionalmente vistos como menos humanos. Deste modo, as pessoas LGBTQ+ padecerão de múltiplas exclusões, como as de expressarem sua existência e de serem presidiários(as). Privados de liberdade, esses sujeitos são submetidos à perpetuação de uma lógica social violenta e opressiva $(1,2,7)$. Há limitação de direitos básicos, destacando-se o direito à saúde, e marcas permanentes de desigualdade, marginalização e precariedade de recursos para construção de políticas públicas que permitam a plena reintegração social.

A partir dessa problemática, este artigo se propõe debater a realidade da garantia da assistência à saúde de pessoas LGBTQ+ enquanto luta pela solidificação dos direitos humanos frente às políticas públicas de saúde no sistema prisional brasileiro.

\section{Metodologia}

Foi realizada análise bibliográfica e documental sobre a temática. Para isso, realizouse um levantamento de artigos e livros que tratavam da discussão histórica acerca da garantia dos direitos humanos das pessoas LGBTQ+, marcando o direito à saúde frente às políticas públicas de saúde no sistema prisional brasileiro. A busca foi realizada em bases 
de dados virtuais, tais como Bireme, Scielo e Lilacs, tendo-se como descritores os termos: direito à saúde, minorias sexuais e de gênero, políticas públicas e sistema prisional, com seus correspondentes em inglês e espanhol. Os critérios de inclusão deram-se para textos publicados em português, espanhol e inglês; cronologicamente a partir do ano 2000; e trabalhos completos disponíveis online. Os critérios de exclusão utilizados foram textos que não contivessem pelo menos três dos descritores concomitantemente; que estivessem sem resumo e sem bibliografia; trabalhos duplicados; artigos que não abordassem a discussão do sistema prisional; e vias teóricas que não possuíssem o mesmo escopo teórico empregado tal qual este trabalho.

Além dos artigos e livros levantados, foram utilizados materiais oficiais da Organização das Nações Unidas (ONU), da Organização Mundial da Saúde (OMS), portarias e decretos do Ministério da Justiça, Secretaria Especial dos Direitos Humanos, Departamento Penitenciário Nacional. Também, foram consultadas a Política Nacional de Atenção Integral à Saúde das Pessoas Privadas de Liberdade no Sistema Prisional; Política Nacional de Atenção Básica; Política Nacional de Saúde Integral de Lésbicas, Gays, Bissexuais, Travestis e Transexuais; orientadas pelas normativas do Sistema Único de Saúde (SUS).

\section{Resultados e discussão}

Em relação ao Sistema Prisional Brasileiro e às existências LGBTQ+, a Resolução conjunta número 1, de 15 de abril 2014 (8), assinada pelo Conselho Nacional de Combate à Discriminação de LGBT (CNCD/LGBT) e pelo Conselho Nacional de Política Criminal e Penitenciária (CNPCP), estabelece tratamento e acolhimento específico a essa população nas instituições prisionais, levando em consideração a segurança e especial vulnerabilidade desses sujeitos. Essa Resolução orienta, entre outras coisas, que as pessoas LGBTQ+ em privação de liberdade devem ter seus registros realizados com seu nome social, a garantia de tratamento hormonal e o acompanhamento médico assegurado dentro do presídio. Além disso, preconiza que eles e elas tenham direito à local de convivência específico e que o uso de roupas masculinas ou femininas seja facultativo para pessoas transexuais (9).

Debater sobre a garantia do direito à saúde no Brasil tem se tornado sinônimo da luta pela solidificação dos direitos humanos. Faz-se importante refletir que, como enfatizado claramente no documento Princípios Básicos para o Tratamento de Prisioneiros, da 
Organização das Nações Unidas (10), quando um Estado tira o direito das pessoas à liberdade, ele assume a responsabilidade de cuidar de sua saúde, tanto em relação às condições do ambiente da instituição, quanto aos tratamentos que podem ser necessários (10). E, no contexto brasileiro, reforça-se que devem ser consideradas as diretrizes de atenção do SUS (11) e as políticas públicas de saúde relacionadas a essa população.

Porém, há omissões do Estado na assistência ao indivíduo privado de liberdade que, acompanhado do encarceramento massivo e crescente no país de populações pobres e pretas, cria descasos e violação de direitos vivenciados nos espaços prisionais, destacandose o descumprimento da assistência sanitária, o que impõe riscos ao direito à saúde (2, 7, 12). Sobral Silva (13) destaca que, como populações vulneráveis nas prisões brasileiras, têm-se as gestantes, lactantes, crianças, idosos, pessoas LGBTQ+, e as negras; todas com demandas específicas de cuidados e atenção em prol da manutenção de suas saúdes.

As condições precárias do sistema de saúde prisional afetam esses grupos em situação de vulnerabilidade. Em 2014, no Brasil, menos da metade (49\%) dos presídios possuíam consultórios médicos (49\%) e consultórios odontológicos (43\%). Além disso, havia uma quantidade de profissionais de saúde insuficiente: um médico para 929 detentos(as) e um enfermeiro para cada 505 privados(as) de liberdade. (14). Sobre as unidades prisionais que apresentavam serviços com foco na população LGBTQ+, em 2014, dos 1.420 presídios no Brasil, 5\% ( $n=73)$ apresentavam aspectos específicos para essa população, e 1\% $(n=$ 10) apresentava ala específica (14).

Assim, há tempos, a precariedade da situação da saúde de pessoas em privação de liberdade, de modo geral, é tema de debates nos meios acadêmicos e nos espaços de formulação e gerenciamento das políticas públicas. Nos últimos anos, o Brasil desenvolveu políticas de saúde específicas para esse público, podendo citar a Lei de Execução Penal (LEP), de 1984; o Plano Nacional de Saúde no Sistema Penitenciário (PNSSP), de 2004; e a Política Nacional de Atenção Integral à Saúde das Pessoas Privadas de Liberdade no Sistema Prisional (PNAISP), de 2014 (15, 16, 17).

A LEP prevê que a assistência à saúde do preso e do internado de caráter preventivo e curativo compreenderá atendimento médico, farmacêutico e odontológico, a toda a população prisional (provisória ou apenada) (15). Nessa lei, observa-se que as nomenclaturas para a população prisional eram preso e condenado, remetendo a uma ideia reducionista do sujeito, uma vez que fica restrito ao ato criminoso cometido (15). 
Entre a LEP e o PNSSP, houve dois marcos legais inestimáveis para a garantia do direito à saúde para as pessoas privadas de liberdade: a Constituição da República Federativa do Brasil (CRFB), de 1988 (18), e a Lei n 8.080, de 19 de setembro de 1990, que institui o SUS (11). Ao longo dessas últimas décadas, houve uma mudança na sensibilidade governamental em relação às pessoas privadas de liberdade, a ponto de se realizar essas e outras normativas no sentido de assegurar o direito à saúde dessa parcela da população brasileira.

O PNSSP, por sua vez, veio legitimar a garantia do acesso à saúde para as pessoas privadas de liberdade, de acordo com o princípio de universalidade do SUS, ampliando as diretrizes de saúde no sistema penitenciário descritas anteriormente na LEP (19). Além disso, observa-se mudança nos termos para identificação da população penitenciária, como os termos pessoas privadas de liberdade, população confinada nas unidades prisionais (16).

O Ministério da Saúde, considerando a orientação sexual e a identidade de gênero como determinantes sociais e as desfavoráveis condições de saúde da população LGBTQ+, e visando a redução das iniquidades e desigualdades sanitárias nesse grupo populacional, elaborou a Política Nacional de Saúde Integral de Gays, Lésbicas, Bissexuais, Travestis e Transexuais (PNSILGBT). Aprovada pelo Conselho Nacional de Saúde, em novembro de 2009, a PNSILGBT teve seu Plano Operativo pactuado na Comissão Intergestores Tripartite (CIT), em novembro de 2011 e foi publicada por meio da Portaria $n^{\circ} 2.836$, de 01 de dezembro de 2011 (20).

Destaca-se ainda que, a PNSILGBT apresenta esforços das três esferas de governo e da sociedade civil organizada na promoção, na atenção e no cuidado em saúde, priorizando a redução das desigualdades por tais identificações sexuais e de gênero, assim como o combate à homofobia, lesbofobia e transfobia, e a discriminação nas instituições e serviços de saúde. Suas diretrizes e seus objetivos estão, portanto, voltados para a promoção da equidade. Além disso, é uma política transversal, com gestão e execução compartilhadas entre as três esferas governamentais, que deverá atuar articulada às demais políticas do Ministério da Saúde (20).

Com o intuito de sintetizar a sequência histórica dos principais momentos e avanços nas políticas de atenção em saúde a população LGBTQ+ que contribuíram para a construção da PNSILGBT, apresenta-se a linha do tempo elaborada por Popadiuk, Oliveira e Signorelli (21), expressa na figura 1. 
Figura 1. Algumas situações-chave que contribuíram para a construção da Política Nacional de Saúde Integral de Gays, Lésbicas, Bissexuais, Travestis e Transexuais de 1988 a 2016

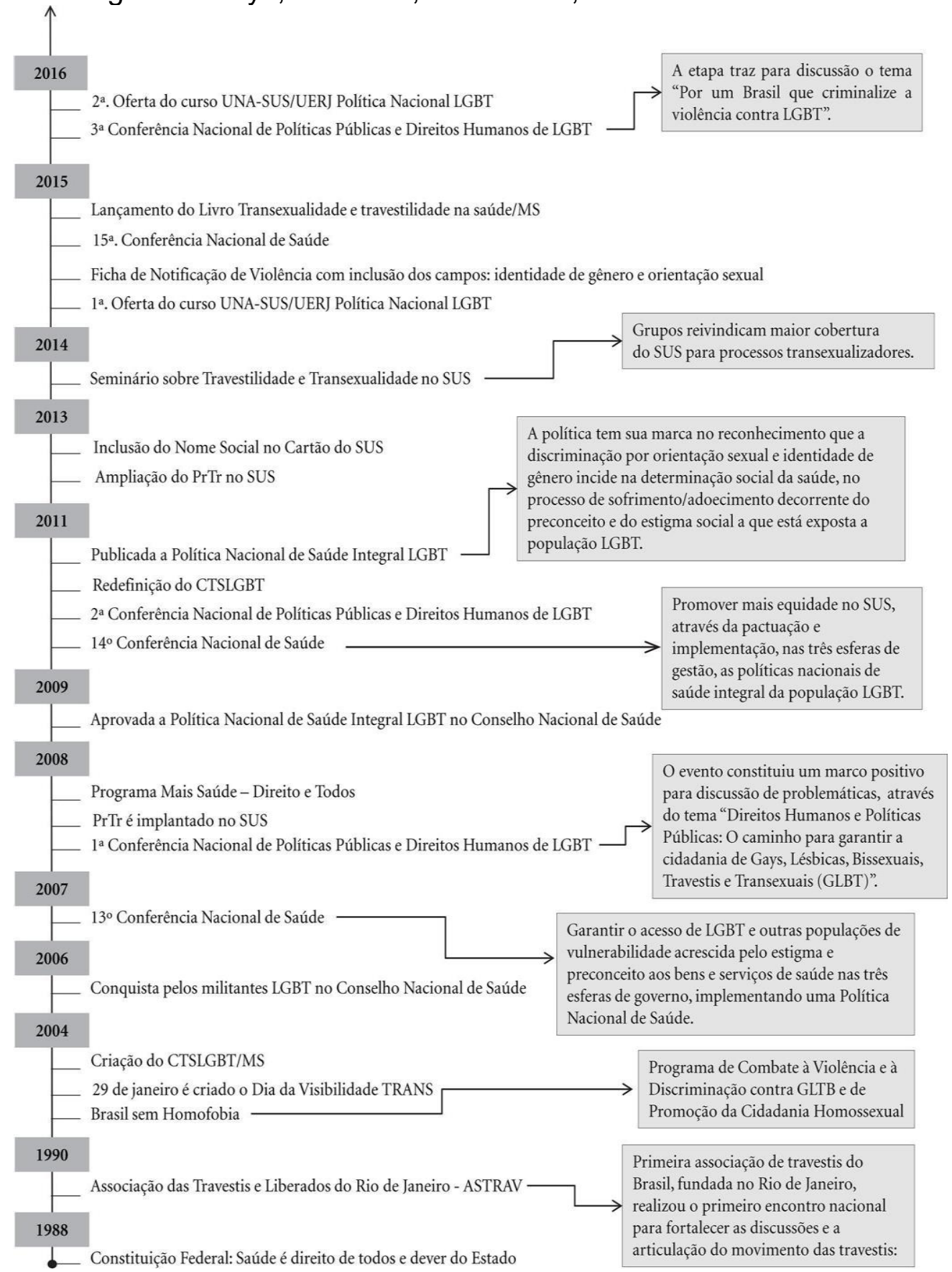

Fonte: Popadiuk, Oliveira e Signorelli (29).

A PNSSP incluiu em suas diretrizes o respeito ao que determina a PNSILGBT e 0 Plano Nacional de Promoção da Cidadania e Direitos Humanos LGBT. Em maio de 2009, o Governo Federal lançou o Plano Nacional de Promoção da Cidadania e Direitos Humanos LGBT, composto por 51 diretrizes, que deveriam ser transformadas em políticas de Estado (22). Esses avanços políticos puderam assegurar a permanente luta pela integralidade e o 
acesso aos serviços de saúde das pessoas LGBTQ+ e ao enfrentamento de atravessamentos dimensões individual, social e programática, que aumentam ou diminuem a vulnerabilidade ao adoecimento delas.

Como marco mais recente, tem-se a PNAISP, a qual visa garantir o acesso integral ao SUS da população privada de liberdade, por meio da qualificação e da humanização da assistência, com ações conjuntas nas áreas da saúde e da justiça, nas esferas federal, estaduais e municipais (22). Nessa Política, o foco é ampliado, tendo como público-alvo todas as pessoas que se encontram sob custódia do Estado, em regime fechado, semiaberto, aberto ou cumprindo medida de segurança na modalidade de tratamento ambulatorial. Além disso, ela impulsiona a criação da Resolução nº 01, de 10 de fevereiro de 2014, a qual dispõe sobre a proteção e os direitos das pessoas com transtornos mentais e redireciona o modelo assistencial em saúde mental, e da Resolução CNCD/LGBT já citada anteriormente $(9,23)$.

A PNAISP prevê que os serviços de saúde no sistema prisional passem a ser um ponto da Rede de Atenção à Saúde (RAS) do SUS, qualificando também a Atenção Primária à Saúde (APS) no âmbito prisional como porta de entrada do sistema e ordenadora das ações e serviços de saúde pela rede. É importante destacar que, quando o estabelecimento prisional não estiver preparado para prover a assistência necessária, deverá provê-la em outro local, sendo necessária para isso a autorização da direção do estabelecimento. As equipes de Atenção Primária à Saúde Prisional (ou Atenção Básica Prisional, como é trazido na Política) apresentam composição multiprofissional e devem realizar suas atividades nas unidades prisionais ou nas unidades básicas de saúde a que estiverem vinculadas. O número de pessoas e o perfil epidemiológico dessas pessoas determinarão as modalidades de equipe (14).

As equipes podem se organizar em cinco modalidades, basicamente sendo: 1) Equipe de Atenção Básica Prisional Tipo I, formada por cinco profissionais com seis horas semanais (enfermeiro, médico, técnico ou auxiliar de enfermagem, cirurgião-dentista e técnico ou auxiliar de saúde bucal); 2) Equipe de Atenção Básica Prisional Tipo I com Saúde Mental, formada por oito profissionais com seis horas semanais (cinco profissionais somados a um psiquiatra ou um médico com experiência em Saúde Mental e dois profissionais escolhido entre terapeuta ocupacional, fisioterapeuta, psicólogo, assistente social, farmacêutico ou enfermeiro); 3) Equipe de Atenção Básica Prisional Tipo II, formada por oito profissionais 
com vinte horas semanais (cinco profissionais somados a um psicólogo, um assistente social e um profissional escolhido entre: terapeuta ocupacional, psicólogo, fisioterapeuta, nutricionista, farmacêutico, assistente social ou enfermeiro); 4) Equipe de Atenção Básica Prisional Tipo II com Saúde Mental, formada por 11 profissionais com vinte horas semanais (cinco profissionais somados a um psiquiatra ou um médico com experiência em Saúde Mental, um psicólogo, um assistente social e três profissionais escolhidos entre: terapeuta ocupacional, psicólogo, fisioterapeuta, nutricionista, farmacêutico, assistente social ou enfermeiro); 5) Equipe de Atenção Básica Prisional Tipo III, formada por 11 profissionais com trinta horas semanais (cinco profissionais, somados a um psiquiatra ou um médico com experiência em Saúde Mental, um psicólogo, um assistente social e três profissionais escolhidos entre: terapeuta ocupacional, psicólogo, fisioterapeuta, nutricionista, farmacêutico, assistente social ou enfermeiro) (14).

Quando o município não adere à política, é importante reforçar a responsabilidade gestora e sanitária, no âmbito da Secretaria Municipal de Saúde, pelo atendimento da população prisional no seu território, em especial nas situações de urgência e emergência, devido ao recebimento dos recursos, de forma regular e automática, pelo incentivo para a atenção à saúde no sistema penitenciário, por meio do Piso da Atenção Básica (PAB) Variável, componente da Política Nacional de Atenção Básica (PNAB), conforme Portaria número 204, de janeiro de 2007 (VI - Incentivo para a Atenção à Saúde no Sistema Penitenciário), e Portaria nํㅜ 2488, de outubro de 2011 e Portaria nํㄴ.436, de 21 de setembro de $2017(23,24,25)$.

Um dos desafios impostos à implantação da PNAISP está na adesão facultativa dos municípios, que alegam que o valor de incentivo repassado pela União é irrisório perante a demanda e o volume de procedimentos requeridos pelas unidades prisionais (26). As entidades governamentais têm perdido oportunidades singulares de manterem o direito à saúde dessa população, adotando postura negligente frente à situação. A OMS considera que uma das mais fortes lições aprendidas no fim do século XX é que a Saúde Pública não pode mais ignorar a saúde da pessoa privada de liberdade. Por isso, existe um crescente reconhecimento de que uma boa atenção à saúde nos sistemas prisionais representa uma boa saúde pública (27). E quando se trata das pessoas LGBTQ+, que apresentam demandas e especificidades na saúde (28), é emergente uma ação que rompa o preconceito e privação dos direitos fundamentais $(29,30)$. 


\section{Conclusão}

Pensar a população LGBTQ+ no espaço prisional é entender as diversas vulnerabilidades e condições que esses indivíduos vivem em ambiente intra e extra prisionais. No sistema de saúde brasileiro, a reflexão é pensar na garantia de atendimento e de acesso à saúde dessa população, entendendo suas especificidades segundo as questões de gênero e as práticas afetivas e sexuais. A saúde é um direito fundamental de todo cidadão e assegurá-la diante das particularidades é entender os atravessamentos sociais, culturais, sexuais e econômicos que perpassam todo sujeito. Afirmar a saúde para esses indivíduos é uma forma de subverter a ordem social que os coloca vulneráveis, por impedi-los de exercerem seus direitos e por questionarem as lógicas da norma heterossexual, as lógicas da acumulação de bens, e as lógicas da submissão perante as violências e opressões no ambiente prisional.

Negar e precarizar o acesso à saúde no sistema carcerário no país, principalmente no atendimento à saúde LGBTQ+, expõe, além da marginalização de suas existências, o descuido (intencional) no atendimento às demandas de saúde, configurando-se uma tríplice carga de sofrimento e privação de direitos: direito de ser quem são; direito de estarem onde estão; direito de receberem o que necessitam.

Assim, faz-se necessário que qualquer ação voltada à saúde de sujeitos LGBTQ+ privados(as) de liberdade a considere uma população historicamente marginalizada, que tem sua existência negada e sua condição de abjeção, que a coloca como vivências periféricas. Dentro do sistema prisional, devem-se considerar aspectos da saúde sexual e reprodutiva, direitos sociais, civis e políticos dessas pessoas. $O$ acesso aos serviços de saúde, tanto nas questões de prevenção quanto nas de tratamento, deve ser assegurado, assim como deve, também, assegurar a autonomia dos indivíduos no tocante à vivência da sua sexualidade e de seu gênero, sem discriminação, negligência ou violência. Assegurar esses aspectos é entender que um princípio fundamental do Estado é, sobretudo, fazer viver.

\section{Referências}

1. Santana NTT, Volpato LMB. O preconceito Enfrentado por Homossexuais Privados de Liberdade. Seminário Integrado. 2015:1-9.

2. Kolling GJ, Silva MB, Pires de Sá MCDN. O direito à saúde no sistema Prisional. Revista Tempus Actas Saúde. 2013;7(1):281-297. 
3. Mombaça J. Rumo a uma redistribuição desobediente de gênero e anticolonial da violência. Oficina de imaginação política. Fundação Bienal de São Paulo, p. 11; 2016.

4. Butler J. Regulações de Gênero. Cadernos Pagu. 2014;42:249-274.

5. Ayres, JRCM, Calazans GJ, Saletti Filho, Haraldo C, França Junior I. Risco, vulnerabilidade e práticas de prevenção e promoção da saúde. In: Tratado de saúde coletiva [S.I: s.n.]; 2009.

6. Butler J. Corpos que pesam: sobre os limites discursivos do sexo. In: Louro GL (org.) O Corpo educado: Pedagogias da Sexualidade. Belo Horizonte: Editora Autêntica; 2002. pp. 151-172.

7. Monteiro FM, Cardoso GR. A Seletividade do sistema prisional brasileiro e o perfil da população carcerária: um debate oportuno. Revista Civitas. 2013;13(1):93-117.

8. Brasil. Resolução n 01, de 10 de fevereiro de 2014. Ministério da Justiça: Brasília; 2014.

9. Brasil. Resolução conjunta CNPCP-CNCD/LGBT, de 15 de abril de 2014. Ministério da Justiça: Brasília; 2014.

10. Organização das Nações Unidas - ONU. Regras Mínimas para Tratamento de Prisioneiros. Genebra: UNESCO; 1955.

11. Brasil. Lei nº 8.080, de 19 de setembro de 1990. Brasília: Diário Oficial da União; 1900.

12. Silva JWSB, Silva Filho CN, Bezerra HMC, Duarte KVN, Quinino LRM. Políticas públicas de saúde voltadas à população LGBT e à atuação do controle social. Espaço para a Saúde. 2017;18(1):140-149.

13. Sobral Silva C. A terceirização de presídios a partir do estudo de uma penitenciária do Ceará. Dezembro. 2005. Disponível em: https://jus.com.br/artigos/6541/a-terceirizacao-depresidios-a-partir-do-estudo-de-uma-penitenciaria-do-ceara. Acesso em: 27 out. 2017.

14. Brasil. Ministério de Justiça. Levantamento Nacional de Informações Penitenciárias INFOPEN, junho de 2014. Brasília: Departamento Penitenciário Nacional; 2014.

15. Brasil. Lei no 7.210, de 11 de julho de 1984. Institui a Lei de Execução Penal. Brasília: Ministério da Justiça; 1984.

16. Brasil. Plano Nacional de Saúde no Sistema Penitenciário. Ministério da Saúde: Brasília; 2004.

17. Brasil. Política Nacional de Atenção Integral à Saúde das Pessoas Privadas de Liberdade no Sistema Prisional. Ministério da Saúde e Ministério da Justiça: Brasília; 2014.

18. Brasil. Constituição da República Federativa do Brasil. Brasília: Diário Oficial da União; 1988. 
19. Lermen HS, Gil BL, Cunico SD, Jesus LO. Saúde no cárcere: análise das políticas sociais de saúde voltadas à população prisional brasileira. Physis Revista de Saúde Coletiva. 2015;25(3):905-924.

20. Brasil. Portaria no 2.836, de $1^{\circ}$ de dezembro de 2011. Institui, no âmbito do Sistema Único de Saúde (SUS), a Política Nacional de Saúde Integral de Lésbicas, Gays, Bissexuais, Travestis e Transexuais (Política Nacional de Saúde Integral LGBT). Brasil. Ministério da Saúde; 2011.

21. Popadiuk GS, Oliveira DC, Signorelli MC. A Política Nacional de Saúde Integral de Lésbicas, Gays, Bissexuais e Transgêneros (LGBT) e o acesso ao

ProcessoTransexualizador no Sistema Único de Saúde (SUS): avanços e desafios. Ciência \& Saúde Coletiva. 2017;22(5):1509-1520.

22. Brasil. Plano Nacional de Promoção da Cidadania e Direitos Humanos de LGBT. Brasília: Secretaria Especial dos Direitos Humanos; 2009.

23. Brasil. Portaria nํ204, de 29 de janeiro de 2007. Brasília: Ministério da Saúde; 2007.

24. Brasil. Portaria no 2.488, de 21 de outubro de 2011. Aprova a Política Nacional de Atenção Básica, estabelecendo a revisão de diretrizes e normas para a organização da Atenção Básica, para a Estratégia Saúde da Família (ESF) e o Programa de Agentes Comunitários de Saúde (PACS). Brasília: Ministério da Saúde; 2011.

25. Brasil. Portaria № 2.436, de 21 de setembro de 2017. Aprova a Política Nacional de Atenção Básica, estabelecendo a revisão de diretrizes para a organização da Atenção Básica, no âmbito do Sistema Único de Saúde (SUS). Brasília: Ministério da Saúde; 2017.

26. Freitas RS, Zermiani TC, Nievola MTS, Nasser JN, Ditterich RG. Política nacional de atenção integral à saúde das pessoas privadas de liberdade no sistema prisional: uma análise do seu processo de formulação e implantação. Revista de Políticas Públicas. 2016;20(1):171-184.

27. Organização Mundial da Saúde - OMS. Health in prisons: a WHO guide the essentials in prison health. Copenhagen, Denmark: WHO; 2007.

28. Albuquerque GA, Garcia CL, Alves MJH, Queiroz CMHT, Adami F. Homossexualidade e o direito à saúde: um desafio para as políticas públicas de saúde no Brasil. Saúde em Debate. 2013;37(98):516-524.

29. Cardoso MR, Ferro LP. Saúde e População LGBT: Demandas e Especificidades em Questão. Psicologia: Ciência e Profissão. 2012;32(3):552-563.

30. Oliveira BP, Silva MAS, Souza MS. O direito à saúde de pessoas trans* no Distrito Federal: entre o direito de existir e o direito à equidade. Cadernos lbero-americanos de Direito Sanitário. 2019;8(1):10-25. 


\section{Colaboradores}

Souza LPS e Minucci GS participaram da concepção do artigo, análise e interpretação dos dados, redação, revisão e aprovação da versão final do texto. Alves AM, Alves RJR e Fernandes MM participaram da análise e interpretação dos dados e da revisão crítica e aprovação da versão final do artigo.

Como citar este artigo:

Souza LPS, Minucci GS, Alves AM, Alves RJR, Fernandes MM. Direito à saúde das pessoas LGBTQ+ em privação de liberdade: o que dizem as políticas sociais de saúde no Brasil?. Cadernos Ibero-Americanos de Direito Sanitário. 2020 abr.jun.; 9(2): 135-148.

http://dx.doi.org/10.17566/ciads.v9i2.582 